\title{
Ocular Injuries Associated With the Use of Airguns
}

\author{
ANTHONY T. MOORE, ALISON McCARTNEY and ROBERT J. COOLING \\ London
}

\begin{abstract}
Summary
Sixty patients with eye injuries resulting from the use of airguns were admitted to a large eye hospital over an 11-year period. The typical patient was a young male teenager; 70 per cent of patients were under the age of 17, the age at which it is legally permissible to own an air weapon. In 4 cases the missile lodged in the ocular adnexae, in 18 cases there was a penetrating eye injury and in 38 patients blunt nonpenetrating eye injury. The prognosis for visual recovery was poor especially following penetration of the globe; visual acuity was reduced to $6 / 60$ or less in 40 per cent of all eyes and in 18 per cent the injured eye was excised.
\end{abstract}

The eye is a common site of injury caused by missiles fired from airguns and the injured eye is often severly damaged with associated loss of vision. In this retrospective study we reviewed all cases of airgun related eye injuries admitted to a large Eye Hospital over an 11-year period. We discuss the types of injury that may occur, their visual prognosis and consider how the incidence of these injuries may be reduced.

\section{Patients and Methods}

The clinical records of all patients with airgun related eye injuries admitted to Moorfields Eye Hospital between October 1974 and October 1985 were reviewed. Patients with similar injuries presenting to the Accident and Emergency Department but not requiring admission were excluded from the study. Histopathological specimens of eyes enucleated following airgun injuries were also reviewed.

\section{Results}

Sixty patients were included in this study; 53 of the injuries were caused by airgun pellets, four by darts, two by the airgun spring, and one by a piece of pear loaded into an airgun and fired at close range. There were 49 males and 11 females whose ages ranged from 13 months to 52 years (mean 17 years) (Fig. 1).

The injuries were divided into 3 groups:

(1) Injuries to the ocular adnexae alone (4 cases).

(2) Penetrating ocular injuries (18 cases).

(3) Blunt (non-penetrating) ocular injuries (38 cases).

\section{Group I: Ocular Adnexae (4 cases)}

In 4 patients there was no injury to the globe; the pellet was lodged in the upper lid in 2 cases and in the subcutaneous tissues of the brow in the remaining two. All patients underwent surgical removal of the pellet and made uneventful recoveries.

\section{Group II: Penetrating Ocular Injuries}

(18 cases)

Of the 18 penetrating eye injuries, 16 were caused by pellets and two by darts. The 2 eyes injured by darts recovered good vision following surgical repair; in both cases the dart had

From: Institute of Ophthalmology and Moorfields Eye Hospital, London.

Correspondence to: A. T. Moore, FRCS, Addenbrookes Hospital, Cambridge.

Presented at the Annual Congress of the Ophthalmological Society of the United Kingdom, April 1986. 


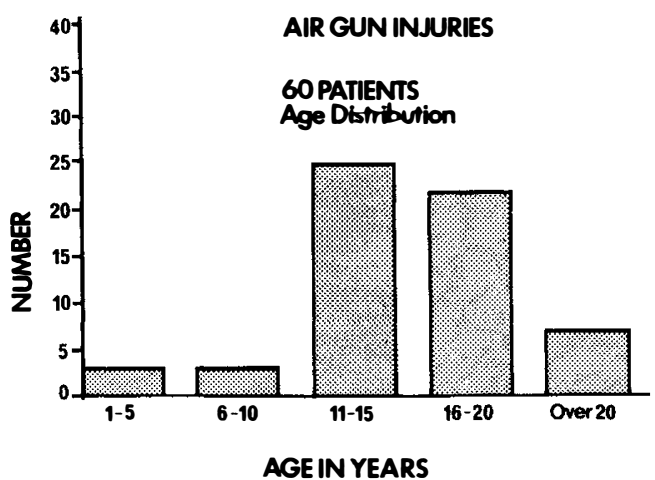

Fig. 1. Airgun injuries: Age range (60 patients).

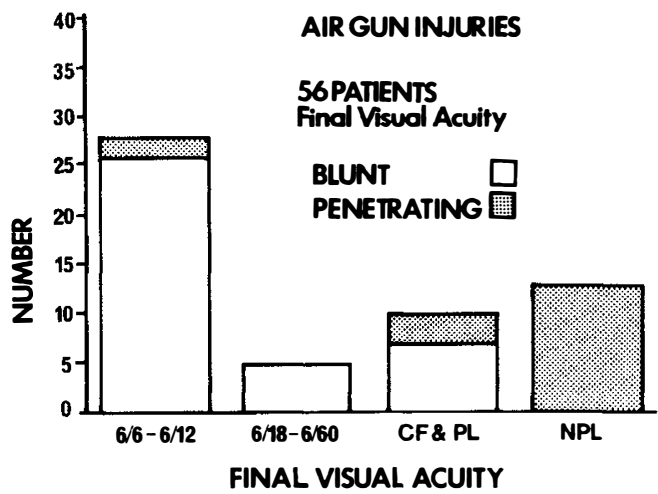

Fig. 2. Airgun injuries: Final visual acuity (56 patients).

traversed the lid and only the tip had penetrated the globe resulting in localised injury.

Penetrating injuries caused by pellets had a poor prognosis. Of the 16 eyes injured by pellets 11 were enucleated and a further two rendered blind; none of the eyes retained visual acuity of better than counting fingers (Fig. 2). Thirteen of the eyes sustained severe injuries with extensive intraocular disorganisation with haemorrhagic retinal detachment; 3 eyes with less extensive injuries developed late rhegmatogenous retinal detachment which in one case was associated with a giant tear. Most patients required more than one operation (Table I).

In seven of the eyes the pellet was retained within the globe and in a further seven within the orbit.

\section{Histopathology}

A total of 7 enucleated eyes were examined at the Institute of Ophthalmology and in all but one there was a corneal entry wound.

As a consequence of the initial impact or in the course of primary repair, 4 eyes were rendered aphakic and 2 had disrupted lenses. The trajectory of the pellet caused amputation of the iris in 2 cases and gross disruption of the ciliary body in 3 cases.

Six of the 7 globes were found to have haemorrhage in the anterior chamber and in 2 cases intraocular contents were prolapsing through the corneal wound. In addition, two other samples of prolapsed tissue were examined. The prolapsed contents in these 4 cases included retina and blood clot in 2 cases and uvea, either iris or iris and choroid, in the remaining 2 cases. Three eyes showed obvious loss of vitreous.

The pellet was retained in 3 eyes following primary repair. These were found to be impacted in the sclera rather than in the centre of the globe with fragmentation of foreign material into the adjacent sclera and some evidence of a foreign body reaction identified in one case 10 days following injury. In one case there was a scleral laceration following ricochet injury; in a further case there was a double penetrating injury of the globe with scleral perforation (Fig. 3).

Haemorrhagic retinal detachment with associated necrosis was the most common sequel to airgun pellet injury seen in 5 cases with coincidental prolapse of the retina in the remaining 2 cases. Vitreous haemorrhage was identified in 3 cases and suprachoroidal haemorrhage in 2 others.

One eye contained a vitreous abscess 8 days following primary repair and 3 eyes showed focal infiltration of the choroid by eosinophils and lymphocytes at 7 days (2 eyes) and 19 days. No evidence of overt sympathetic granulomatous inflammation was identified.

\section{Table I Penetrating ocular injuries (18 eyes)}

$\begin{array}{ll}\text { Surgical Procedures } & \\ \text { Primary enucleation } & 1 \\ \text { Primary repair alone } & 2 \\ \text { Primary repair/enucleation } & 9 \\ \text { Exploratory vitrectomy/enucleation } & 1 \\ \text { Primary repair/vitrectomy } & 4 \\ \text { Primary repair/cataract surgery/detachment } & \\ \text { surgery } & 1\end{array}$




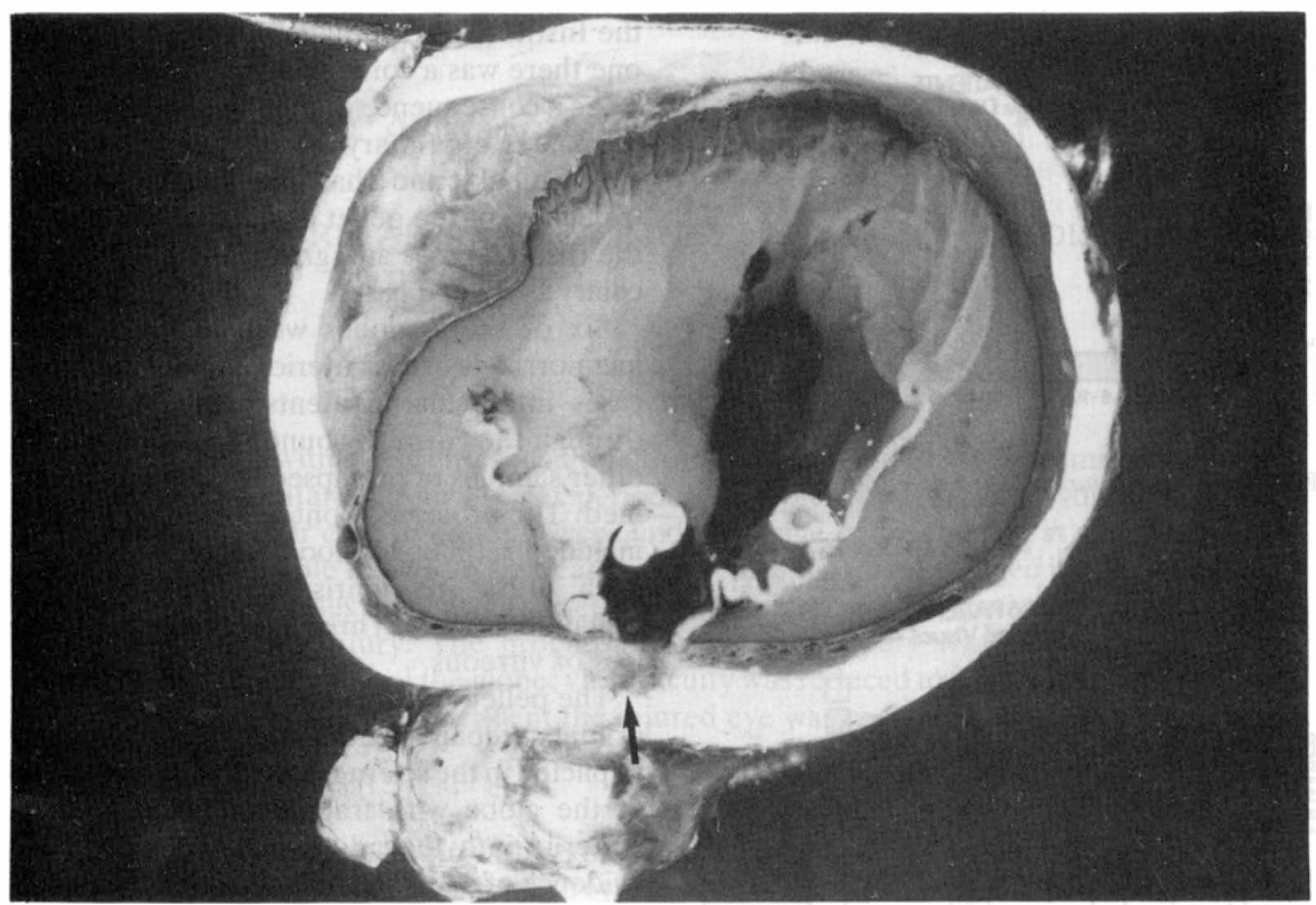

Fig. 3. Histological specimen of enucleated eye showing total retinal detachment, posterior scleral perforation (arrow) and retina lying outside the sclera.

The pattern of damage in these eyes suggests two distinct mechanisms may be responsible. If the pellet enters cleanly, without tumbling, the trajectory appears to predispose to amputation injuries of the anterior segment with loss of the lens and subsequent embedding of the pellet within the sclera (or double penetration). Such eyes tended to show less suprachoroidal haemorrhage and gross disruption of the retina than those eyes where the lens was shattered and the pellet presumed to have tumbled and caused severe haemorrhagic disruption of the retina. Prolapse of intraocular contents appears to occur more readily with this type of injury.

All eyes suffered considerable posterior segment structural damage and although only 3 eyes showed evidence of possible early sympathetic uveitis, this complication must be considered a potential risk of such injuries.

\section{Group III: Blunt (non-penetrating) Ocular Injuries (38 cases)}

Of the 38 eyes with a contusional injury 33 were caused by pellets, 2 by darts, 2 by the spring of an air rifle and one by a piece of pear. Thirteen pellets and 2 darts were localised within the orbit ( 2 pellets and 2 darts were later removed at surgery). A wide variety of anterior and posterior segment damage was evident (Table II). Six of the eyes developed retinal detachment; four were rhegmatogenous, one a traction detachment and one a localised haemorrhagic detachment. Two of the rhegmatogenous detachments developed secondary to a dialysis and two in association with a macular break.

Overall blunt trauma resulted in less severe injury and a better visual prognosis (Fig. 2). Sixty-eight per cent of injured eyes retained $6 / 12$ vision or better. In 7 eyes the final visual acuity was less than $6 / 60$; in 2 cases this followed optic nerve injury and in the remaining 5 macular scarring or macular pucker. No eyes were excised.

Fewer patients in this group required surgery (Table III).

The following 2 cases illustrate the type of injury seen in this group. 
Table II Blunt injury (38 eyes)

\begin{tabular}{lr}
\hline Anterior Segment & \\
Hyphaema & 24 \\
Iridodialysis & 7 \\
Cataract & 7 \\
Dislocated lens & 3 \\
Glaucoma & 1 \\
Posterior Segment & \\
Vitreous haemorrhage & 18 \\
Commotio retinae & 23 \\
Macular hole & 3 \\
Macular pucker & 3 \\
Choroidal rupture & 13 \\
Retinal detachment & 6 \\
Other injuries & \\
Optic nerve injury & 2 \\
Orbital cellulitis & 2 \\
Medial rectus paresis & 1 \\
\hline
\end{tabular}

Table III Blunt injury (38 eyes)

\begin{tabular}{ll}
\hline Surgical Procedures & \\
Vitreoretinal surgery & 4 \\
Cataract surgery & 4 \\
Lateral orbitotomy & 2 \\
EUA & 2 \\
\hline
\end{tabular}

\section{Case I}

A 12-year-old child was accidentally shot in the right eye by a friend and was examined immediately at the local eye department and transferred to Moorfields Eye Hospital for further management.

On admission, visual acuity was $3 / 60$ in the right eye and $6 / 5$ in the left. There was a small entry wound of the right lower lid, extensive subconjunctival haemorrhage and microscopic hyphaema. A right relative afferent pupil defect was discovered in the presence of extensive intra-retinal haemorrhage and commotio retinae. There was a large direct choroidal rupture inferiorly and a fullthickness macular break with no associated retinal detachment. Orbital X-rays confirmed the presence of a retained airgun pellet.

He was discharged from hospital 2 days following admission and over the ensuing 3 months vision improved to $6 / 36$ in the right eye. Extensive choroid-retinal scarring was visible at the site of impact.

Ten months following the injury he developed further visual loss in the right eye and was found to have a subtotal retinal detachment with a macular break and a small oral tear adjacent to the impact site. Closed vitrectomy was undertaken with internal drainage of subretinal fluid and fluid/gas exchange performed. The macular break was sur-

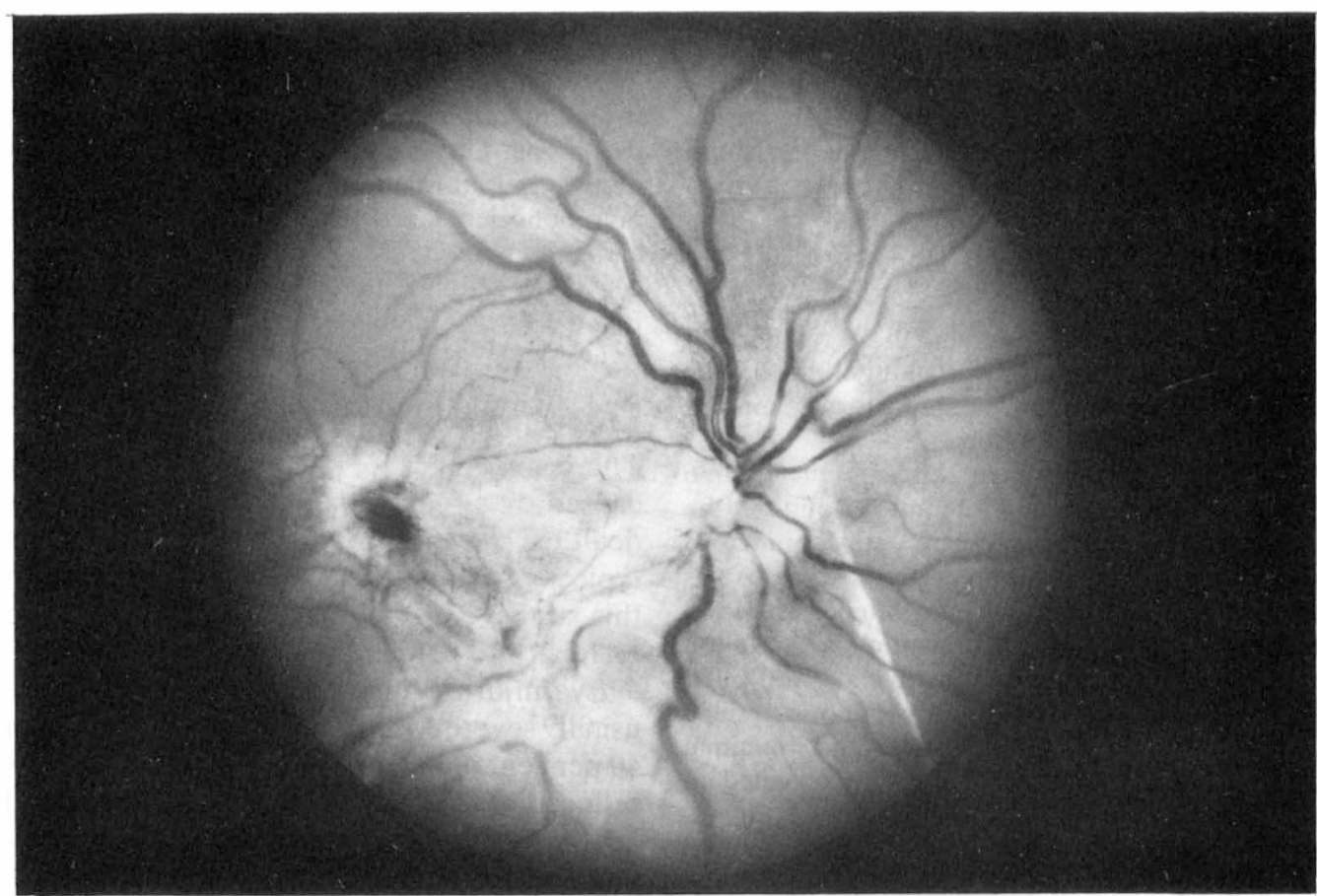

Fig. 4. Case I: Right fundus showing macular pucker following blunt injury caused by impact of airgun pellet. 


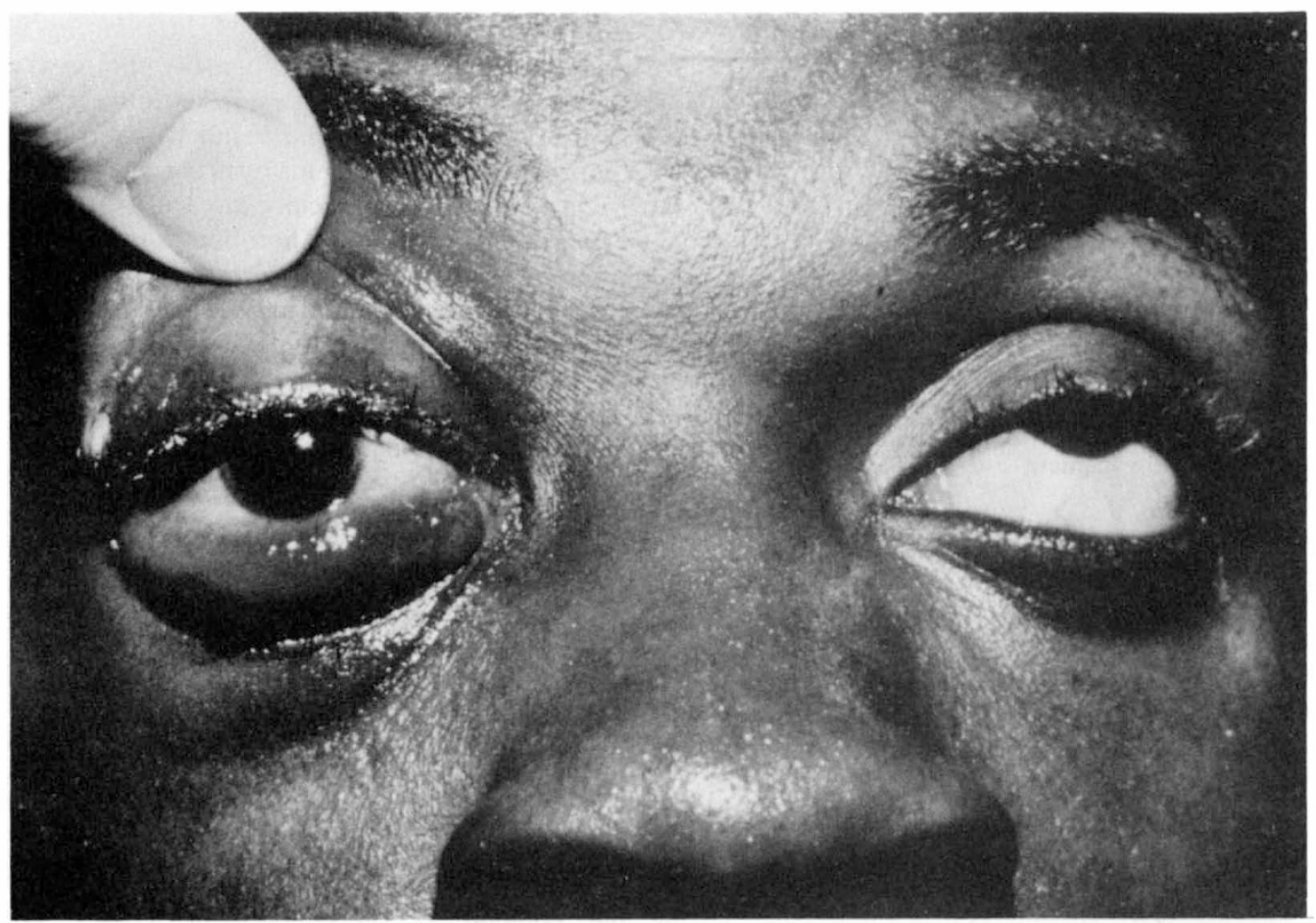

Fig. 5. Case II: Right proptosis and limited upgaze following airgun injury.

rounded by endolaser photocoagulation and the peripheral tear sealed by trans-scleral cryotherapy and supported on a circumferential explant. The retina was successfully reattached and the visual acuity restored to $6 / 36$. Subsequently development of an epimacular membrane (Fig. 4) reduced vision to counting fingers.

\section{Case II}

A 20-year-old man who was shot in the right eye by an unknown assailant while walking in the street. He was immediately aware of blurred vision in this eye, the left eye being amblyopic.

On admission vision was $6 / 24$ in the right and $1 / 36$ in the left. There was a $4 \mathrm{~mm}$ entry wound in the right upper lid with extensive subconjunctival haemorrhage and associated ptosis. There was axial proptosis measuring $6 \mathrm{~mm}$ with no restriction of ocular movements apart from minimal limitation of elevation. There was no relative afferent pupil defect. There was widespread commotio retinae with intraretinal haemorrhages and minimal vitreous haemorrhage. Orbital X-rays revealed an airgun dart located at the right orbital apex (Fig. 6). Broad spectrum antibiotics were administered systemically.

Over the next few days there was increasing pro- ptosis with marked restriction of ocular movements in all directions (Fig. 5). Nine days following the injury a right lateral orbitotomy was carried out revealing an intraorbital abscess surrounding the dart. The abscess was drained and the dart removed, gram staining revealing abundant polymorphonuclear leucocytes but no micro-organisms and sterile culture. Two weeks following surgery vision had returned to $6 / 9$ and ocular motility was full apart from mild limitation of abduction of the right eye (Figs 5 and 6 ).

\section{Discussion}

There have been several reports of injuries caused by airguns ${ }^{1-7}$ and a few of these have dealt exclusively with ocular injuries. ${ }^{1,6.7}$ The typical patient is a young male teenager; in this study 80 per cent of those injured were male and 70 per cent under the age of 17 .

Eye injuries caused by airgun missiles are usually severe with a poor visual prognosis; in 40 per cent of patients in this series vision was reduced to $6 / 60$ or less in the injured eye and in 18 per cent the extent of the injury necessitated enucleation. The most severe injuries were found in the penetrating injury group in 


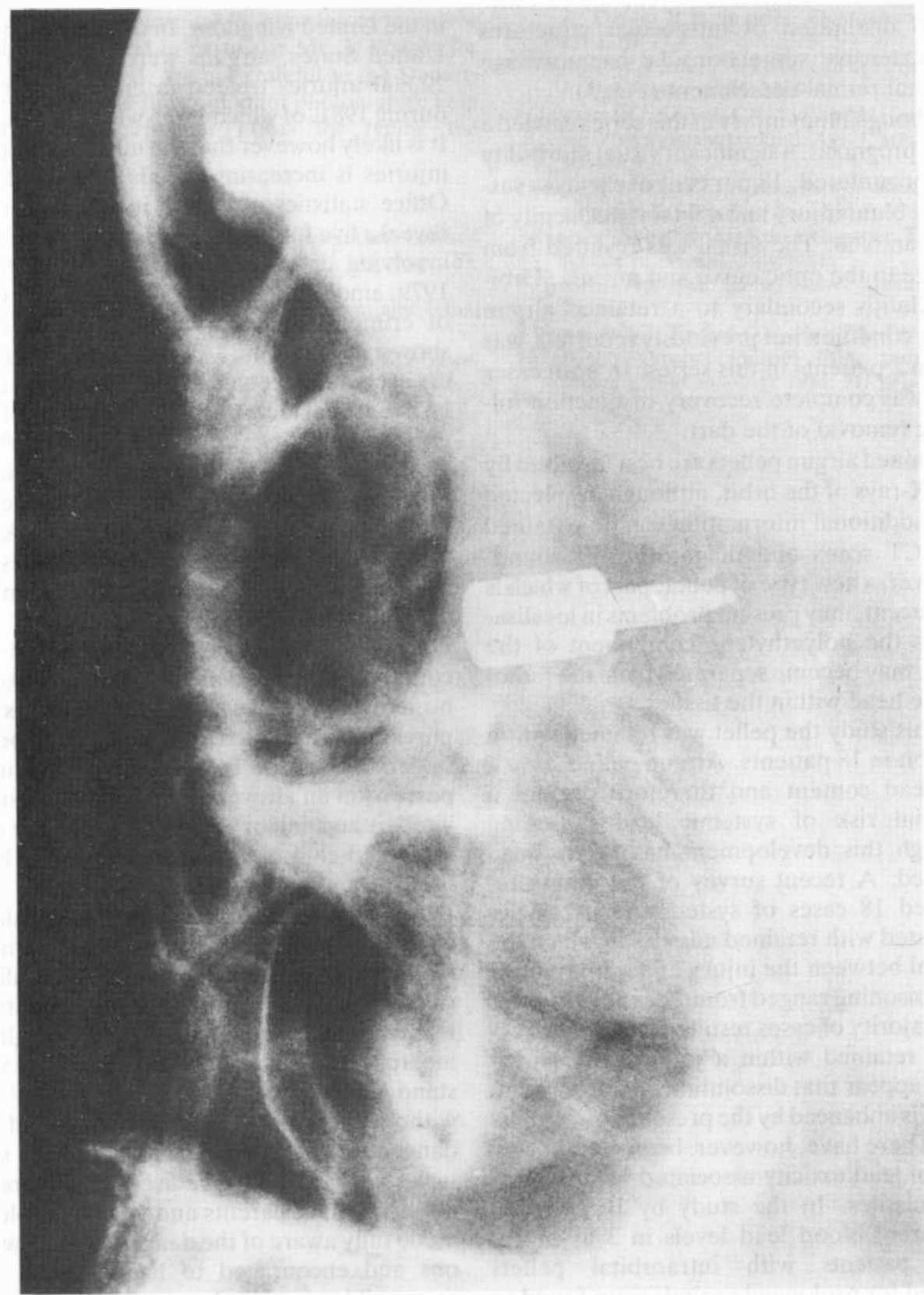

Fig. 6. Case II: Plain X-ray of orbit showing retained airgun dart. 
which none of the eyes penetrated by an airgun pellet retained useful vision and most were ultimately excised. Histopathological examination of enucleated eyes showed widespread disruption of intraocular structures with extensive suprachoroidal haemorrhage and total retinal detachment (Fig. 3).

Although blunt injury in this series carried a better prognosis, a significant visual morbidity was encountered; 18 per cent of the eyes sustaining blunt injury had a final visual acuity of less than $6 / 60$. The visual loss resulted from damage to the optic nerve and macula. Orbital cellulitis secondary to a retained airgun dart, a condition not previously reported, was seen in 2 patients in this series. In both cases there was complete recovery of function following removal of the dart.

Retained airgun pellets are best localised by plain X-rays of the orbit, although in selected cases additional information can be obtained from CT scans and diagnostic ultrasound. However, a new type of pellet, part of which is radiolucent, may present problems in localisation as the polyethylene component of the missile may become separated from the radioopaque head within the tissues. ${ }^{9}$

In this study the pellet was retained within the orbit in 18 patients. Airgun pellets have a high lead content and therefore present a potential risk of systemic lead poisoning although this development has rarely been reported. A recent survey of the literature ${ }^{8}$ revealed 18 cases of systemic lead toxicity associated with retained missiles in which the interval between the injury and symptoms of lead poisoning ranged from 2 days to 40 years. The majority of cases resulted from lead fragments retained within a joint cavity and it would appear that dissolution and absorption of lead is enhanced by the presence of synovial fluid. There have, however, been no reported cases of lead toxicity associated with intraorbital missiles. In the study by Bowen and Magauren $^{1}$ blood lead levels in 3 asymptomatic patients with intraorbital pellets retained for prolonged periods were found to be within normal limits. Whilst airgun pellets retained within the orbit are best left alone, orbital airgun darts should be removed since in our experience the synthetic fibres sur- rounding the metal tip may induce a severe orbital inflammatory response.

There are no available figures on the incidence of ocular injuries caused by air weapons in the United Kingdom. In one study from the United States, airguns were responsible for 22,800 injuries treated in emergency rooms during 1981, of which 1,255 were eye injuries. ${ }^{6}$ It is likely however that the numbers of airgun injuries is increasing in this country; Home Office statistics of crimes involving firearms reveal a five fold increase in crimes of violence involving air weapons over the period 1969 to 1979 , amounting to 2,773 cases in 1979 . Cases of criminal damage involving air weapons show a similar trend..$^{10}$ The majority of offenders are young teenagers and there appears to be a seasonal increase in the incidence of such crimes coinciding with school holidays. ${ }^{10}$

In considering the prevention of accidents involving air weapons, the legal aspects of airgun ownership are of importance. A firearm certificate is not required for air rifles with a muzzle energy of less than 12 footpounds or an air pistol generating less than 6 footpounds. Most airguns purchased in this country fall within these standards. However, no individual under the age of 17 years may purchase or hire such a weapon and no person under the age of 14 years may have in his possession an air weapon except when supervised by an adult or when he is a member of an approved club or when the weapon is being used at a shooting gallery. ${ }^{11}$ Evidence from Home Office statistics suggest that legislative changes to raise the age at which it is permissible to handle an air weapon would be unlikely to reduce significantly the availability of weapons to young people. ${ }^{10}$ Safety glasses incorporating polycarbonate lenses will withstand the impact of an airgun pellet, ${ }^{12}$ but without greater public awareness of the dangers of these weapons, such safety spectacles are unlikely to be used. It is therefore important that parents and young people are made fully aware of the dangers of such weapons and encouraged to handle them in a responsible manner. A report ${ }^{1}$ in 1973 emphasised that education of the public on the dangers of airguns represented the only approach to reducing the incidence of such injuries. However, there does not appear to 
have been any significant decline in the incidence of these serious injuries.

We would like to thank the Consultant surgeons of Moorfields Eye Hospital for allowing us to review their patients and in particular Mr. J. Wright for details of Case II. We are grateful to the Department of Medical Illustration for photographic help and to Miss Heather Lucas for typing the manuscript.

\section{References}

${ }^{1}$ Bowen DI, Magauran DM: Ocular injuries caused by airgun pellets: an analysis of 105 cases. $\mathrm{Br}$. Med. J. 1973; 1: 333-7.

${ }^{2}$ Rawson HD: The air rifle-a potentially lethal weapon. NZ Med. J. 1965; 64: 327-9.

${ }^{3}$ Reid IS: Airgun injuries in children. Med. J. Aust. 1974; 1: 64-6.
${ }^{4}$ Spitz L: Air rifle injuries in children. S. Afr. Med.J. 1969; 43, 1: 557-60.

${ }^{5}$ Batch AJ: The air rifle: a dangerous weapon. $\mathrm{Br}$. Med. J. 1981; 286: 283.

${ }^{6}$ Stenberg P, De Juan E, Green E, Hurst L, Sommer A: Ocular B.B. injuries. Ophthalmology 1984; 91: 1269-77.

${ }^{7}$ Young DW, Little, JM: Pellet gun eye injuries. Can. J. Ophthamol. 1985; 20: 9-10.

${ }^{8}$ Dillman RO, Crumb CK, Lidsky MJ: Lead poisoning from a gunshot wound. Am. J. Med. 1979; 66: 509-14.

${ }^{9}$ Cain D, Weeks RF: A new danger associated with airgun pellet injuries. Br. Med. J. 1983; 286: 21 .

${ }^{10}$ Shooting Sports Trust: Research paper. The use and misuse of airguns. July 1981.

"Firearms Act 1968: Stones Justice Manual 1982; 1: 2334-74.

${ }^{12}$ Simmons ST, Krohel GB, Hay PB: Prevention of ocular gunshot injuries using polycarbonate lenses. Ophthalmology 1984; 91: 977-84. 